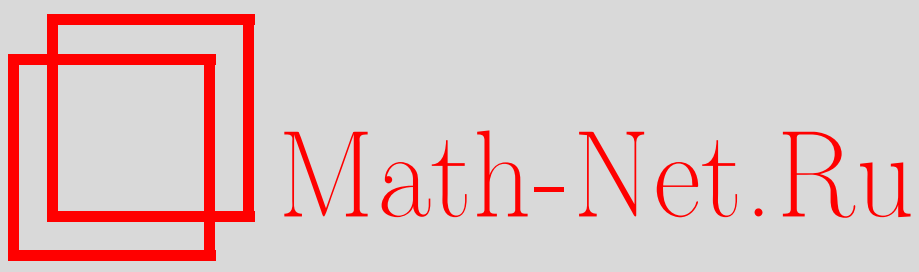

В. П. Михайлов, О существовании граничного значения у бигармонических функций, Матем. сб., 2004, том 195, номер 12, 81-94

DOI: https://doi.org/10.4213/sm866

Использование Общероссийского математического портала Math-Net.Ru подразумевает, что вы прочитали и согласны с пользовательским соглашением

http://www . mathnet.ru/rus/agreement

Параметры загрузки:

IP : 52.87 .193 .239

26 апреля 2023 г., 08:18:56 
УДК 517.928

\author{
В.П. Михайлов
}

\title{
О существовании граничного значения у бигармонических функций
}

\footnotetext{
В работе устанавливаются критерии существования $L_{2}$-предела и слабого $L_{2}$-предела бигармонической функции на правильной аналитической границе двумерной ограниченной области.

Библиограффия: 7 названий.
}

Пусть $Q$ - ограниченная двумерная область, $Q \subset \mathbb{R}_{2}, x=\left(x_{1}, x_{2}\right)$ - точка области $Q, z=\left(x_{1}+i x_{2}\right)$ - ее комплексная координата, и пусть $u(x)=u(z)-$ бигармоническая в области $Q$ функция, т.е. функция, являющаяся решением уравнения

$$
\Delta^{2} u=0, \quad x \in Q
$$

Границу $\partial Q$ области $Q$ будем предполагать правильной аналитической кривой, т.е. кривой такой, что для любой точки $z_{0} \in \partial Q$ существует такая ее (комплексная) окрестность $U_{z_{0}}$, что уравнение попавшего в эту окрестность куска $\partial Q \cap U_{z_{0}}$ кривой $\partial Q$ имеет вид $z=\lambda(t), t_{1}<t<t_{2}$, где $\lambda(t)-$ аналитическая функция вещественного переменного $t \in\left(t_{1}, t_{2}\right), z_{0}=\lambda\left(t_{0}\right), t_{0} \in\left(t_{1}, t_{2}\right), t_{1}=t_{1}\left(z_{0}\right)$, $t_{2}=t_{2}\left(z_{0}\right)$, причем $\lambda^{\prime}(t) \neq 0$ для $t \in\left(t_{1}, t_{2}\right)$.

Обозначим через

$$
w=w(z), \quad z \in Q,
$$

функцию, осуществляющую конформное отображение области $Q$ комплексной $z$-плоскости на единичный круг $\{|w|<1\}$ комплексной $w$-плоскости, а через

$$
z=z(w), \quad|w|<1
$$

обратную к ней функцию. Прообраз при преобразовании (2) кольца $\left\{r_{0}<|w|<1\right\}$ при некотором $r_{0} \in(0,1)$ обозначим через $Q_{r_{0}}$. (Ниже число $r_{0}$ будет выбрано конкретно.) Область $Q_{r_{0}}$ состоит из точек кривых $\gamma_{r}, r_{0}<r<1$, являющихся прообразами окружностей $\{|w|=r\}$ :

$$
\gamma_{r}=\left\{z=z\left(r e^{i \varphi}\right), 0 \leqslant \varphi \leqslant 2 \pi\right\}, \quad r_{0}<r<1 .
$$

Работа вьполнена при поддержке Российского фонда фундаментальных исследований (грант № 01-01-00988) и Программы поддержки ведущих научных школ РФ (грант №

(C) В. П. Михайлов 2004 
Прообразом окружности $\{|w|=1\}$ является граница $\partial Q$ области $Q$, которую естественно по аналогии с (4) обозначить через $\gamma_{1}$,

$$
\partial Q=\gamma_{1}=\left\{z=z\left(e^{i \varphi}\right), 0 \leqslant \varphi \leqslant 2 \pi\right\} .
$$

Пусть в области $Q_{r_{0}}$ задана некоторая непрерывная функция $g(z)$ (в частности, исследуемая бигармоническая функция $u(z)$ ). Будем говорить, что функция $g(z)$ имеет $L_{2}$-предел на границе $\partial Q=\gamma_{1}$, если семейство ее следов на кривых $\gamma_{r}$, $r_{0}<r<1$, т.е. множество функций

$$
\left\{g\left(z\left(r e^{i \varphi}\right)\right), 0 \leqslant \varphi \leqslant 2 \pi\right\}, \quad r_{0}<r<1,
$$

имеет $L_{2}(0,2 \pi)$-предел при $r \rightarrow 1-0$. Если множество функций $(5)$ имеет слабый $L_{2}(0,2 \pi)$-предел при $r \rightarrow 1-0$, то скажем, что функция $g(z)$ имеет слабый $L_{2}$-предел на границе $\partial Q$. Будем также говорить, что функция $g(z) L_{2}$-компактна (слабо $L_{2}$-компактна) вблизи границы $\partial Q$, если множество ее следов $(5) L_{2}(0,2 \pi)$ компактно (соответственно слабо $L_{2}(0,2 \pi)$-компактно). Аналогично скажем, что функция $g(z) L_{2}$-ограничена вблизи границы $\partial Q$, если множество ее следов $(5)$ $L_{2}(0,2 \pi)$-ограничено. Ясно, что свойства функции $g(z)$ быть слабо $L_{2}$-компактной и $L_{2}$-ограниченной вблизи гранищы эквивалентны.

Целью настоящей работы является установление критериев существования $L_{2}$-предела и слабого $L_{2}$-предела бигармонической функции $u(z)$ на границе области $Q$. А именно будет установлена справедливость следуюших утверждений.

Теорема 1. Для того чтобы бигармоническая функиия $u(z), z \in Q$, имела $L_{2}$-предел на граниче области, необходимо и достаточно, чтобъ она была $L_{2}$-компактна вблизи ее граничы.

ТЕОРема 2. Для того чтобъ бигармоническая функиия $u(z), z \in Q$, имела слабьй $L_{2}$-предел на граниче области, необходимо и достаточно, чтобы она была слабо $L_{2}$-компактна вблизи ее границы.

Для случая, когда двумерная область есть круг, теорема 1 и некоторые другие критерии существования $L_{2}$-предельных значений на границе области, причем не только для бигармонических, но и $m$-гармонических функций при любом $m \geqslant 1$, были установлены в работе [1]. Аналогичные результаты были получены в работе [2] для случая полосы в $\mathbb{R}_{n}$ при любом $n \geqslant 2$ для решений линейного эллиптического уравнения с постоянными коэффициентами порядка $2 m, m \geqslant 1$, характеристический многочлен которых отличается от характеристического многочлена для $m$-гармонического оператора на произвольный многочлен степени, менњшей чем $2 m$.

Отметим, что критерии, содержащиеся в теоремах 1 и 2 , относятся к любому решению уравнения (1), в том числе и к произвольной гармонической функции. Для гармонических функций, как известно (см. [3] и [4], а для решений общих линейных эллиптических уравнений второго порядка с достаточно гладкими коэффициентами в ограниченных областях $n$-мерного пространства с достаточно гладкой границей см. [5]), критерием сушествования $L_{2}$-предела на границе является 
условие их принадлежности классу Харди $H_{2}$, т.е. в определенном смысле условие их $L_{2}$-ограниченности вблизи границы. Это, конечно, не противоречит теореме 1 , поскольку для решений эллиптических уравнений второго порядка условие их $L_{2}$-ограниченности вблизи границы является условием их $L_{2}$-компактности. С другой стороны, как показано в работах [1] и [2], условие $L_{2}$-ограниченности вблизи границы для всех решений уравнения порядка, большего чем 2 , критерием быть не может: это условие, конечно, необходимо, но, вообще говоря, достаточным не является.

Заметим, что и в настоящей работе, так же как и в перечисленных вьше работах, предельное значение функции на границе области понимается как предельное значение множества следов этой функции на некоторой системе "параллельных" границе и в определенном смысле аппроксимирующих границу кривых (в многомерном случае - на системе поверхностей). Здесь в качестве такой системы кривых взято множество кривых $\left\{\gamma_{r}\right\}$. Вопрос о возможности замены системы кривых $\left\{\gamma_{r}\right\}$ другими аппроксимирующими границу системами кривых будет рассмотрен нами в другом месте. Отметим лишш, что глубокие результаты в этом направлении для решений эллиптических уравнений второго порядка имеются в работе [6].

Поскольку из $L_{2}$-компактности (или $L_{2}$-слабой компактности) вблизи границы комплекснозначной бигармонической функции $u(z)$, очевидно, вытекает $L_{2}$-компактность (соответственно $L_{2}$-слабая компактность) вблизи границы вешественнозначных бигармонических функций $\operatorname{Re} u(z)$ и $\operatorname{Im} u(z)$, то сформулированные выше теоремы достаточно доказать для вешественнозначных бигармонических функций. В связи с этим в дальнейшем мы будем считать функцию $u(z)$ принимаюшей лишь вешественные значения.

Хорошо известно [7], что для любой вешественнозначной бигармонической в области $Q$ функции $u(z)$ сушествуют две аналитические в $Q$ функции $f_{1}(z)$ и $f_{2}(z)$ комплексного переменного $z$ такие, что

$$
u(z)=\operatorname{Re}\left(f_{1}(z)+\bar{z} f_{2}(z)\right), \quad z \in Q .
$$

Функции $f_{1}$ и $f_{2}$ определяются по функции $u(z)$ с точностью до линейных слагаемых. Точнее, функция $f_{1}(z)$ определяется с точностью до слагаемого $\alpha z+a i$, a функция $f_{2}(z)$ - с точностью до слагаемого $i b z-\bar{\alpha}$, где $\alpha$ - произвольное комплексное число, а $a$ и $b-$ произвольные вещественные числа. Доказательство этого утверждения состоит в проверке того, что равенству

$$
\operatorname{Re}\left(g_{1}(z)+\bar{z} g_{2}(z)\right) \equiv 0, \quad z \in Q,
$$

в котором $g_{1}(z)$ и $g_{2}(z)$ - аналитические в $Q$ функции, удовлетворяют только функции $g_{1}(z)=\alpha z+a i$ и $g_{2}(z)=i b z-\bar{\alpha}$ при указанных выше $\alpha, a$ и $b$. То, что эти функции равенству (7) удовлетворяют, очевидно:

$$
\operatorname{Re}(\alpha z+a i+\bar{z}(z b i-\bar{\alpha}))=\operatorname{Re}\left(\alpha z+i b|z|^{2}-\overline{z \alpha}\right)=\operatorname{Re}(\alpha z-\overline{z \alpha}) \equiv 0 .
$$

Для доказательства обратного утверждения подставим в (7) $g_{1}(z)=u_{1}(x)+$ $i v_{2}(x), g_{2}(z)=u_{2}(x)+i v_{2}(x)$, где $u_{k}$ и $v_{k}, k=1,2,-$ соответствующие вещественнозначные гармонические в $Q$ функции. В результате равенство $(7)$ заменится эквивалентным равенством

$$
u_{1}(x)+x_{1} u_{2}(x)+x_{2} v_{2}(x) \equiv 0, \quad x \in Q .
$$


После применения к (8) оператора Лапласа получим тождество

$$
u_{2 x_{1}}(x)+v_{2 x_{2}}(x) \equiv 0, \quad x \in Q
$$

которое в силу условий Коши-Римана для функции $g_{2}(z)$ приводит к равенствам $u_{2}(x)=c_{0} x_{2}+c_{1}, v_{2}(x)=-c_{0} x_{1}+c_{2}$, т.е. $g_{2}(z)=-c_{0} i z+c_{1}+i c_{2}$, где $c_{0}, c_{1}, c_{2}-$ произвольные вещественные постоянные. Подставляя найденные значения для $u_{2}$ и $v_{2}$ в $(8)$, получаем, что $u_{1}(x) \equiv-c_{1} x_{1}-c_{2} x_{2}$ и, тем самым, согласно условиям Коши-Римана для функции $g_{1}(z)$ имеем $v_{1}(x)=-c_{1} x_{2}+c_{2} x_{1}+c_{3}$, где $c_{3}$ - вешественная постоянная, т.е. $g_{1}(z)=z\left(-c_{1}+i c_{2}\right)+i c_{3}$. Что и требовалось установить (при этом $\left.\alpha=-c_{1}+i c_{2}, a=c_{3}, b=-c_{0}\right)$.

Поскольку добавление к функциям $f_{1}(z)$ и $f_{2}(z)$ линейных слагаемых не изменяет их поведения вблизи границы области в интересующем нас смысле, т.е. в смысле существования предельного значения на границе области у функции $u(z)$ или ее $L_{2}$-компактности (или ее слабой $L_{2}$-компактности) вблизи границы, то в дальнейшем мы ограничимся каким-либо конкретным выбором в равенстве (6) этих функций.

В результате преобразования (2) равенство (6) приобретает вид

$$
U(w)=\operatorname{Re}\left(F_{1}(w)+\overline{z(w)} F_{2}(w)\right), \quad|w|<1
$$

где $U(w)=u(z(w)),|w|<1,-$ вещественнозначная функция, а $F_{1}(w)=f_{1}(z(w))$ и $F_{2}(w)=f_{2}(z(w))$ - аналитические в круге $\{|w|<1\}$ функции.

В силу принятого вьше определения исследуемая бигармоническая функция $u(z)$ имеет $L_{2}$-предел или слабый $L_{2}$-предел на границе $\partial Q$ тогда и только тогда, когда семейство функций

$$
\left\{U\left(r e^{i \varphi}\right), 0 \leqslant \varphi \leqslant 2 \pi\right\}, \quad r_{0}<r<1
$$

имеет $L_{2}(0,2 \pi)$-предел или соответственно слабый $L_{2}(0,2 \pi)$-предел при $r \rightarrow 1-0$. Аналогично, $L_{2}$-компактность или слабая $L_{2}$-компактность (т.е. $L_{2}$-ограниченность) функции $u(z)$ вблизи границы $\partial Q$ эквивалентны $L_{2}(0,2 \pi)$-компактности или соответственно слабой $L_{2}(0,2 \pi)$-компактности (т.е. $L_{2}(0,2 \pi)$-ограниченности) семейства функций (10). Следовательно, доказательство сформулированных теорем 1 и 2 эквивалентно доказательству соответствующих утверждений для функции $U(w)$, т.е. доказательству следующих теорем $1^{\prime}$ и $2^{\prime}$.

Теорема 1'. Для того чтобы множсество функиий (10) имело $L_{2}(0,2 \pi)$ предел при $r \rightarrow 1-0$, необходимо и достаточно, чтобъ это мнохсество было $L_{2}$-компактным.

ТЕОРема 2'. Для того чтобы множество функиий (10) имело слабый $L_{2}(0,2 \pi)$-предел при $r \rightarrow 1-0$, необходимо и достаточно, чтобъ это множсество было слабо $L_{2}(0,2 \pi)$-компактным, т.е. $L_{2}(0,2 \pi)$-ограниченным.

В силу предположения о границе $\partial Q$ области $Q$ функция $z(w),|w|<1$, из (3) аналитически продолжается в круг $\{|w|<R\}$ некоторого радиуса $R=R(\partial Q)>1$, 
и, тем самым, функция $\overline{z(1 / \bar{w})}$ является аналитической по $w$ для $|w|>1 / R$. Поскольку нас интересует поведение функции $U\left(r e^{i \varphi}\right), 0 \leqslant \varphi \leqslant 2 \pi, r<1$, для $r$ достаточно близких к $r=1$, то наряду с равенством (9) для функции $U(w)$ можно воспользоваться также вытекающим из него, но справедливым лишш в кольце $\{1 / R<|w|<1\}$ равенством

$U(w)=\operatorname{Re}\left(\left(F_{1}(w)+\overline{z\left(\frac{1}{\bar{w}}\right)} F_{2}(w)\right)+\left(\overline{z(w)}-\overline{z\left(\frac{1}{\bar{w}}\right)}\right) F_{2}(w)\right), \quad \frac{1}{R}<|w|<1$.

Отметим, что в качестве числа $r_{0}$, которое было введено при рассмотрении семейства кривых $\gamma_{r}(4)$, можно взять любое число из полуинтервала $[1 / R, 1)$; в дальнейшем будем считать $r_{0}=1 / R$.

Функция $\left.F_{1}(w)+\overline{z(1 / \bar{w}}\right) F_{2}(w)$ является аналитической функцией переменного $w$ в кольце $\{1 / R<|w|<1\}$. Пусть ее лорановское разложение в этом кольце имеет ВИД

$$
F_{1}(w)+\overline{z\left(\frac{1}{\bar{w}}\right)} F_{2}(w)=G_{1}(w)+\widetilde{G}_{1}(w), \quad \frac{1}{R}<|w|<1
$$

где

$$
G_{1}(w)=\sum_{k=0}^{\infty} a_{k} w^{k}, \quad|w|<1
$$

- правильная часть ряда Лорана, а

$$
\widetilde{G}_{1}(w)=\sum_{k=1}^{\infty} \frac{\widetilde{a}_{k}}{w^{k}}, \quad|w|>\frac{1}{R}
$$

- главная его часть; функции $G_{1}(w)$ и $\widetilde{G}_{2}(w)$ аналитичны соответственно в круге $\{|w|<1\}$ и вне круга $\{|w| \leqslant 1 / R\}$. Таким образом, для функции $U(w)$ окончательно получаем равенство

$$
U(w)=U_{0}(w)+\operatorname{Re}\left(G_{1}(w)+A(w) G_{2}(w)\right), \quad \frac{1}{R}<|w|<1,
$$

в котором

$$
U_{0}(w)=\operatorname{Re}\left(\widetilde{G}_{1}(w)\right), \quad|w|>\frac{1}{R},
$$

- гармоническая в $\{|w|>1 / R\}$ функция, имеющая на окружности $\{|w|=1\}$ $L_{2}$-предельное значение, поскольку эта окружность принадлежит множеству $\{|w|>1 / R\}$, функции $G_{1}(w)$ и $G_{2}(w)$ аналитичны в круге $\{|w|<1\}$, а функция

$$
A(w)=\overline{z(w)}-\overline{z\left(\frac{1}{\bar{w}}\right)}, \quad \frac{1}{R}<|w|<R
$$

как функция вещественных переменных $w_{1}$ и $w_{2}\left(w=w_{1}+i w_{2}=r e^{i \varphi}\right)$ бесконечно диффреренцируема в кольце $\{1 / R<|w|<R\}, A(w) \in C^{\infty}\left(1 / R^{2}<w_{1}^{2}+w_{2}^{2}<R^{2}\right)$, причем

$$
\left.A\left(r e^{i \varphi}\right)\right|_{r=1}=\left.\left(\overline{z\left(r e^{i \varphi}\right)}-\overline{z\left(\frac{1}{r} e^{i \varphi}\right)}\right)\right|_{r=1}=0, \quad 0 \leqslant \varphi \leqslant 2 \pi
$$


Множество функций (10), которое будет предметом нашего исследования, в связи с равенством (12) порождает соответствующие множества функций, отвечающие функциям $U_{0}(w), \operatorname{Re}\left(G_{1}(w)\right), \operatorname{Re}\left(A(w) G_{2}(w)\right)$, т.е. множества

$$
\begin{array}{ll}
\left\{U_{0}\left(r e^{i \varphi}\right), 0 \leqslant \varphi \leqslant 2 \pi\right\}, & \frac{1}{R}<r<1, \\
\left\{\operatorname{Re} G_{1}\left(r e^{i \varphi}\right), 0 \leqslant \varphi \leqslant 2 \pi\right\}, & \frac{1}{R}<r<1, \\
\left\{\operatorname{Re}\left(A\left(r e^{i \varphi}\right) G_{2}\left(r e^{i \varphi}\right)\right), 0 \leqslant \varphi \leqslant 2 \pi\right\}, & \frac{1}{R}<r<1 .
\end{array}
$$

Отметим, что, как уже вьше было сказано, множество функций (13) $L_{2}(0,2 \pi)$ компактно и при $r \rightarrow 1$ имеет $L_{2}(0,2 \pi)$-предел.

Пусть

$$
G_{2}(w)=\sum_{k=0}^{\infty} b_{k} w^{k}, \quad|w|<1
$$

и

$$
z(w)=\sum_{k=0}^{\infty} c_{k} w^{k}, \quad|w|<R
$$

суть тейлоровские разложения функций $G_{1}(w)$ и $z(w)$.

Для коэффициентов $a_{k}, b_{k}$ и $c_{k}, k \geqslant 0$, разложений $(11),(16)$ и (17) (как хорошо известно) справедливы оценки:

для любого $\rho_{1}<1$

$$
\left|a_{k}\right| \leqslant \frac{M_{1}\left(\rho_{1}\right)}{\rho_{1}^{k}}, \quad M_{1}\left(\rho_{1}\right)=\max _{|w|=\rho_{1}}\left|G_{1}(w)\right|, \quad k \geqslant 0
$$

для любого $\rho_{2}<1$

$$
\left|b_{k}\right| \leqslant \frac{M_{2}\left(\rho_{2}\right)}{\rho_{2}^{k}}, \quad M_{2}\left(\rho_{2}\right)=\max _{|w|=\rho_{2}}\left|G_{2}(w)\right|, \quad k \geqslant 0
$$

для любого $\rho_{3}<R$

$$
\left|c_{k}\right| \leqslant \frac{M_{3}\left(\rho_{3}\right)}{\rho_{3}^{k}}, \quad M_{3}\left(\rho_{3}\right)=\max _{|w|=\rho_{3}}|z(w)|, \quad k \geqslant 0
$$

Из (11), (16) и (17) при $1 / R<|w|<1$ имеем равенство

$$
G_{1}(w)+A(w) G_{2}(w)=\sum_{k=0}^{\infty} a_{k} w^{k}+\sum_{k=0}^{\infty} \bar{c}_{k}\left(\bar{w}^{k}-\frac{1}{w^{k}}\right) \sum_{k=0}^{\infty} b_{k} w^{k}
$$


из которого вытекает, что при любом $r, 1 / R<r<1$,

$$
\begin{aligned}
\left(G_{1}(w)+\right. & \left.A(w) G_{2}(w)\right)\left.\right|_{w=r e^{i \varphi}} \\
= & \sum_{k=0}^{\infty} a_{k} r^{k} e^{i k \varphi}+\sum_{k=0}^{\infty} \bar{c}_{k}\left(r^{k}-r^{-k}\right) e^{-i k \varphi} \sum_{s=0}^{\infty} b_{s} r^{s} e^{i s \varphi} \\
= & \sum_{k=0}^{\infty} a_{k} r^{k} e^{i k \varphi}+\sum_{-\infty}^{\infty} e^{i k \varphi}\left(\sum_{m=1}^{\infty} \bar{c}_{m} b_{k+m}\left(r^{m}-r^{-m}\right) r^{m+k}\right) \\
= & \sum_{k=0}^{\infty}\left(a_{k} r^{k}+\sum_{m=1}^{\infty} \bar{c}_{m} b_{k+m}\left(r^{m}-r^{-m}\right) r^{m+k}\right) e^{i k \varphi} \\
& +\sum_{k=1}^{\infty} e^{-i k \varphi} \sum_{m=k}^{\infty} \bar{c}_{m} b_{m-k}\left(r^{m}-r^{-m}\right) r^{m-k} \\
= & \sum_{k=0}^{\infty}\left(a_{k} r^{k}+\sum_{m=1}^{\infty} \lambda_{m k}\left(r^{m}-r^{-m}\right) r^{m+k}\right) e^{i k \varphi} \\
& +\sum_{k=1}^{\infty}\left(\sum_{m=k}^{\infty} \mu_{m k}\left(r^{m}-r^{-m}\right) r^{m-k}\right) e^{-i k \varphi}
\end{aligned}
$$

где

$$
\begin{aligned}
& \lambda_{m k}=\bar{c}_{m} b_{m+k}, \quad k \geqslant 0, \quad m \geqslant 1, \\
& \mu_{m k}=\bar{c}_{m} b_{m-k}, \quad k \geqslant 1, \quad m \geqslant k .
\end{aligned}
$$

Поэтому

$$
\begin{aligned}
\operatorname{Re}\left(G_{1}(w)\right. & \left.+A(w) G_{2}(w)\right)\left.\right|_{w=r e^{i \varphi}} \\
= & \sum_{k=1}^{\infty}\left(a_{k}^{\prime} r^{k}+\sum_{m=1}^{\infty} \lambda_{m k}^{\prime}\left(r^{m}-r^{-m}\right) r^{m+k}\right. \\
& \left.+\sum_{m=k}^{\infty} \mu_{m k}^{\prime}\left(r^{m}-r^{-m}\right) r^{m-k}\right) \cos k \varphi \\
& -\sum_{k=1}^{\infty}\left(a_{k}^{\prime \prime} r^{k}+\sum_{m=1}^{\infty} \lambda_{m k}^{\prime \prime}\left(r^{m}-r^{-m}\right) r^{m+k}\right. \\
& \left.-\sum_{m=k}^{\infty} \mu_{m k}^{\prime \prime}\left(r^{m}-r^{-m}\right) r^{m-k}\right) \sin k \varphi \\
& +a_{0}^{\prime}+\sum_{m=1}^{\infty} \lambda_{m 0}^{\prime}\left(r^{m}-r^{-m}\right) r^{m}
\end{aligned}
$$

где $a_{k}^{\prime}, \lambda_{m k}^{\prime}, \mu_{m k}^{\prime}$ - вешественные части соответствуюших чисел $a_{k}, \lambda_{m k}, \mu_{m k}, \mathrm{a}$ $a_{m}^{\prime \prime}, \lambda_{m k}^{\prime \prime}, \mu_{m k}^{\prime \prime}-$ мнимые части этих чисел. Поскольку

$r^{m}-r^{-m}=\left(r-r^{-1}\right)\left(r^{m-1}+r^{m-3}+\cdots+r^{-(m-1)}\right)=-\left(1-r^{2}\right) \sum_{s=0}^{m-1} r^{m-2-2 s}$, 
то последнее равенство удобно переписать в виде

$$
\begin{aligned}
\operatorname{Re}\left(G_{1}(w)\right. & \left.+A(w) G_{2}(w)\right)\left.\right|_{w=r e^{i \varphi}} \\
= & \sum_{k=1}^{\infty}\left(a_{k}^{\prime} r^{k}-\left(1-r^{2}\right) \sum_{m=1}^{\infty} \lambda_{m k}^{\prime} \sum_{s=0}^{m-1} r^{2 m-2-2 s+k}\right. \\
& \left.-\left(1-r^{2}\right) \sum_{m=k}^{\infty} \mu_{m k}^{\prime} \sum_{s=0}^{m-1} r^{2 m-2-2 s-k}\right) \cos k \varphi \\
& -\sum_{k=1}^{\infty}\left(a_{k}^{\prime \prime} r^{k}-\left(1-r^{2}\right) \sum_{m=1}^{\infty} \lambda_{m k}^{\prime \prime} \sum_{s=0}^{m-1} r^{2 m-2-2 s+k}\right. \\
& \left.+\left(1-r^{2}\right) \sum_{m=k}^{\infty} \mu_{m k}^{\prime \prime} \sum_{s=0}^{m-1} r^{2 m-2-2 s-k}\right) \sin k \varphi \\
& +\left(a_{0}^{\prime}-\left(1-r^{2}\right) \sum_{m=1}^{\infty} \lambda_{m 0}^{\prime} \sum_{s=0}^{m-1} r^{2 m-2-2 s}\right)
\end{aligned}
$$

Для доказательства сформулированных теорем нам понадобятся несколько вспомогательных утверждений.

Лемма 1. Семейство функиий $(10)\left(r_{0}=1 / R\right) L_{2}(0,2 \pi)$-ограничено тогда и только тогда, когда $L_{2}(0,2 \pi)$-ограничены каждое из семейств функиий (14) $u(15)$.

ДокАзАтЕльство. Эта лемма показывает, что в представлении функции $U(w)$ с помощью равенства (12) три слагаемых правой части этого равенства в определенном смысле независимы по отношению к $L_{2}(0,2 \pi)$-ограниченности: из $L_{2}(0,2 \pi)$ ограниченности их суммы вытекает $L_{2}(0,2 \pi)$-ограниченность каждого слагаемого (напомним, что первое слагаемое $L_{2}(0,2 \pi)$-ограничено, поскольку функция $U_{0}(w)$ непрерывна в области $\{|w|>1 / R\})$. Заметим, что представление (9) функции $U(w)$ этим свойством не обладает: из $L_{2}(0,2 \pi)$-ограниченности суммы не вытекает, вообще говоря, $L_{2}(0,2 \pi)$-ограниченность каждого слагаемого его правой части.

Поскольку множество функций (13), связанное с первым слагаемым равенства $(12), L_{2}(0,2 \pi)$-ограничено, то в доказательстве нуждается лишш утверждение леммы о необходимости.

Пусть множество функций (10) $L_{2}(0,2 \pi)$-ограничено. Тогда сушествует такая постоянная $M>0$, что для всех $r \in(1 / R, 1)$ имеет место неравенство

$$
M \geqslant\left.\int_{0}^{2 \pi}\left(U(w)-U_{0}(w)\right)^{2}\right|_{w=r e^{i \varphi}} d \varphi+\left.\int_{0}^{2 \pi}\left(\operatorname{Re}\left(G_{1}(w)+A(w) G_{2}(w)\right)\right)^{2}\right|_{w=r e^{i \varphi}} d \varphi
$$

которому в силу (22) можно придать вид

$$
\begin{aligned}
M \geqslant & \pi \sum_{k=1}^{\infty}\left(a_{k}^{\prime} r^{k}-\left(1-r^{2}\right) \sum_{m=1}^{\infty} \lambda_{m k}^{\prime} \sum_{s=0}^{m-1} r^{2 m-2-2 s+k}\right. \\
& \left.-\left(1-r^{2}\right) \sum_{m=k}^{\infty} \mu_{m k}^{\prime} \sum_{s=0}^{m-1} r^{2 m-2-2 s-k}\right)^{2}
\end{aligned}
$$




$$
\begin{aligned}
& +\pi \sum_{k=1}^{\infty}\left(a_{k}^{\prime \prime} r^{k}-\left(1-r^{2}\right) \sum_{m=1}^{\infty} \lambda_{m k}^{\prime \prime} \sum_{s=0}^{m-1} r^{2 m-2-2 s+k}\right. \\
& \left.+\left(1-r^{2}\right) \sum_{m=k}^{\infty} \mu_{m k}^{\prime \prime} \sum_{s=0}^{m-1} r^{2 m-2-2 s-k}\right)^{2} \\
& +2 \pi\left(a_{0}^{\prime}-\left(1-r^{2}\right) \sum_{m=1}^{\infty} \lambda_{m 0}^{\prime} \sum_{s=0}^{m-1} r^{2 m-2 s-2}\right)^{2}
\end{aligned}
$$

Следовательно, при любом $N>0$ для всех $r \in(1 / R, 1)$ имеет место неравенство

$$
\begin{aligned}
\sum_{k=1}^{N}\left(a_{k}^{\prime} r^{k}-\left(1-r^{2}\right)\left(\sum_{m=1}^{\infty} \lambda_{m k}^{\prime} \sum_{s=0}^{m-1} r^{2 m-2-2 s+k}\right.\right. \\
\left.\left.\quad+\sum_{m=k}^{\infty} \mu_{m k}^{\prime} \sum_{s=0}^{m-1} r^{2 m-2-2 s-k}\right)\right)^{2} \\
+\sum_{k=1}^{N}\left(a_{k}^{\prime \prime} r^{k}-\left(1-r^{2}\right)\left(\sum_{m=1}^{\infty} \lambda_{m k}^{\prime \prime} \sum_{s=0}^{m-1} r^{2 m-2-2 s+k}\right.\right. \\
\left.\left.\quad-\sum_{m=k}^{\infty} \mu_{m k}^{\prime \prime} \sum_{s=0}^{m-1} r^{2 m-2-2 s-k}\right)\right)^{2} \\
+2\left(a_{0}^{\prime}-\left(1-r^{2}\right) \sum_{m=1}^{\infty} \lambda_{m 0}^{\prime} \sum_{s=0}^{m-1} r^{2 m-2 s-2}\right)^{2} \leqslant \frac{M}{\pi}
\end{aligned}
$$

В силу (21) и неравенств (18)-(20)

$$
\begin{array}{ll}
\left|\lambda_{m k}^{\prime}\right|=\left|\operatorname{Re} \lambda_{m k}\right| \leqslant\left|c_{m}\right|\left|b_{m+k}\right| \leqslant \frac{M_{3}\left(\rho_{3}\right)}{\rho_{3}^{m}} \frac{M_{2}\left(\rho_{2}\right)}{\rho_{2}^{m+k}}, \quad m \geqslant 1, \quad k \geqslant 1, \\
\left|\lambda_{m k}^{\prime \prime}\right| \leqslant \frac{M_{3}\left(\rho_{3}\right)}{\rho_{3}^{m}} \frac{M_{2}\left(\rho_{2}\right)}{\rho_{2}^{m+k}}, & m \geqslant 1, \quad k \geqslant 0, \\
\left|\mu_{m k}^{\prime}\right|=\left|\operatorname{Re} \mu_{m k}\right| \leqslant\left|c_{m}\right|\left|b_{m-k}\right| \leqslant \frac{M_{3}\left(\rho_{3}\right)}{\rho_{3}^{m}} \frac{M_{2}\left(\rho_{2}\right)}{\rho_{2}^{m-k}}, \quad & m \geqslant k \geqslant 0, \\
\left|\mu_{m k}^{\prime \prime}\right| \leqslant \frac{M_{3}\left(\rho_{3}\right)}{\rho_{3}^{m}} \frac{M_{2}\left(\rho_{2}\right)}{\rho_{2}^{m-k}}, & m \geqslant k \geqslant 1 .
\end{array}
$$

Положим

$$
\rho_{3}=\frac{R+1}{2}, \quad \rho_{2}=\frac{R+3}{2(R+1)}
$$

при этом, очевидно,

$$
1<\rho_{3}<R, \quad \rho_{2} \rho_{3}=\frac{R+3}{4}>1
$$


Тогда для всех $r \in(1 / R, 1)$ при любом $k \geqslant 1$

$$
\begin{gathered}
\sum_{m=1}^{\infty}\left|\lambda_{m k}^{\prime}\right| \sum_{s=0}^{m-1} r^{2 m-2-2 s+k} \leqslant \sum_{m=1}^{\infty} \frac{M_{3}\left(\rho_{3}\right) M_{2}\left(\rho_{2}\right)}{\rho_{3}^{m} \rho_{2}^{m+k}} m r^{-k} \\
\leqslant M_{3}\left(\rho_{3}\right) M_{2}\left(\rho_{2}\right) r^{-k} \rho_{2}^{-k} \sum_{m=1}^{\infty} \frac{m}{\rho_{2}^{m} \rho_{3}^{m}} \\
=M_{3}\left(\rho_{3}\right) M_{2}\left(\rho_{2}\right)\left(\frac{R}{\rho_{2}}\right)^{k} \frac{\rho_{2} \rho_{3}}{\left(1-\rho_{2} \rho_{3}\right)^{2}} \leqslant C R^{2 k} \\
\sum_{m=k}^{\infty}\left|\mu_{m k}^{\prime}\right| \sum_{s=0}^{m-1} r^{2 m-2-2 s-k} \leqslant \sum_{m=k}^{\infty} \frac{M_{3}\left(\rho_{3}\right) M_{2}\left(\rho_{2}\right)}{\rho_{3}^{m} \rho_{2}^{m-k}} m r^{-k} \\
=M_{2}\left(\rho_{2}\right) M_{3}\left(\rho_{3}\right) \rho_{2}^{k} r^{-k} \sum_{m=k}^{\infty} \frac{m}{\rho_{2}^{m} \rho_{3}^{m}} \\
=M_{2}\left(\rho_{2}\right) M_{3}\left(\rho_{3}\right)\left(R \rho_{2}\right)^{k} \frac{\rho_{2} \rho_{3}}{\left(1-\rho_{2} \rho_{3}\right)^{2}} \leqslant C R^{k},
\end{gathered}
$$

где

$$
C=\frac{M_{2}\left(\rho_{2}\right) M_{3}\left(\rho_{3}\right)}{\left(1-\rho_{2} \rho_{3}\right)^{2}} \rho_{2} \rho_{3}
$$

при

$$
\rho_{2}=\frac{R+3}{2(R+1)}, \quad \rho_{3}=\frac{R+1}{2} .
$$

Аналогично,

$$
\begin{aligned}
& \sum_{m=1}^{\infty}\left|\lambda_{m k}^{\prime \prime}\right| \sum_{s=0}^{m-1} r^{2 m-2-2 s+k} \leqslant C R^{2 k}, \\
& \sum_{m=k}^{m-1}\left|\mu_{m k}^{\prime \prime}\right| \sum_{s=0}^{m-1} r^{2 m-2-2 s-k} \leqslant C R^{k}
\end{aligned}
$$

и

$$
\sum_{m=1}^{\infty}\left|\lambda_{m 0}^{\prime}\right| \sum_{s=0}^{m-1} r^{2 m-2 s-2} \leqslant C .
$$

Поэтому после перехода к пределу при $r \rightarrow 1-0$ в неравенстве (23) (в котором $1 \leqslant k \leqslant N)$ получим неравенство

$$
\sum_{k=1}^{N}\left(a_{k}^{\prime 2}+a_{k}^{\prime \prime 2}\right)+2{a_{0}^{\prime 2}}^{2} \leqslant \frac{M}{\pi}
$$

справедливое при любом $N>0$, т.е. неравенство

$$
2{a_{0}^{\prime 2}}^{2}+\sum_{k=1}^{\infty}\left(a_{k}^{\prime 2}+a_{k}^{\prime 2}\right) \leqslant \frac{M}{\pi}
$$


из которого вытекает справедливое при всех $r \leqslant 1$ неравенство

$$
\left.\int_{0}^{2 \pi}\left(\operatorname{Re} G_{1}(w)\right)^{2}\right|_{w=r e^{i \varphi}} d \varphi=\pi \sum_{k=1}^{\infty}\left(\left(a_{k}^{\prime} r^{k}\right)^{2}+\left(a_{k}^{\prime \prime} r^{k}\right)^{2}\right)+2 \pi r a_{0}^{\prime 2} \leqslant M
$$

т.е. $L_{2}(0,2 \pi)$-ограниченность семейства функций (14). Согласно равенству (12) $L_{2}(0,2 \pi)$-ограниченность семейства функций (15) есть следствие условия леммы, $L_{2}(0,2 \pi)$-ограниченности семейства $(13)$ и только что доказанной $L_{2}(0,2 \pi)$-ограниченности семейства функций (14). Лемма доказана.

Имеют место также следующие утверждения.

Лемма 2. Пусть семейство функиий (14) $L_{2}(0,2 \pi)$-ограничено. Тогда при $r \rightarrow 1-0$ это семейство имеет $L_{2}(0,2 \pi)$-предел.

Лемма 3. Пусть семейство функиий (15) $L_{2}(0,2 \pi)$-ограничено. Тогда при $r \rightarrow 1-0$ это семейство слабо $L_{2}(0,2 \pi)$-сходится $к$ нулю.

ДокаЗАТЕЛьство ЛЕммы 2. Поскольку $\operatorname{Re} G_{1}(w)$ является в круге $\{|w|<1\}$ гармонической функцией, то справедливость утверждения леммы 2 можно получить, как об этом уже говорилось выше, с помощью результатов работ [3], [4] или [5]. Однако и непосредственное доказательство леммы 2 не представляет трудностей (отметим, что нас интересует лишш вопрос об $L_{2}$-сходимости, в то время как в [3] и [4] изучен более общий случай $L_{p}$-сходимости).

Из (11) имеем при всех $\varphi \in(0,2 \pi)$ и всех $r \in(1 / R, 1)$ равенство

$$
\operatorname{Re} G_{1}\left(r e^{i \varphi}\right)=\sum_{k=0}^{\infty} r^{k}\left(a_{k}^{\prime} \cos k \varphi-a_{k}^{\prime \prime} \sin k \varphi\right)
$$

$a_{k}^{\prime}=\operatorname{Re} a_{k}, a_{k}^{\prime \prime}=\operatorname{Im} a_{k}, a_{k}, k \geqslant 0,-$ коэффициенты разложения в $(11)$, и при всех $\varphi \in[0,2 \pi]$ и $r_{1}, r_{2}$ из интервала $(1 / R, 1)$ равенство

$$
\operatorname{Re} G_{1}\left(r_{1} e^{i \varphi}\right)-\operatorname{Re} G_{1}\left(r_{2} e^{i \varphi}\right)=\sum_{k=1}^{\infty}\left(r_{1}^{k}-r_{2}^{k}\right)\left(a_{k}^{\prime} \cos k \varphi-a_{k}^{\prime \prime} \sin k \varphi\right) .
$$

Таким образом,

$$
\begin{gathered}
\int_{0}^{2 \pi}\left(\operatorname{Re} G_{1}\left(r e^{i \varphi}\right)\right)^{2} d \varphi=2 \pi{a_{0}^{\prime}}^{2}+\pi \sum_{k=1}^{\infty}\left(a_{k}^{\prime 2}+a_{k}^{\prime \prime 2}\right) r^{2 k}, \quad r \in\left(\frac{1}{R}, 1\right) \\
\int_{0}^{2 \pi}\left(\operatorname{Re} G_{1}\left(r_{1} e^{i \varphi}\right)-\operatorname{Re} G_{1}\left(r_{2} e^{i \varphi}\right)\right)^{2} d \varphi \\
=\pi \sum_{k=1}^{\infty}\left(a_{k}^{\prime 2}+a_{k}^{\prime \prime 2}\right)\left(r_{1}^{k}-r_{2}^{k}\right)^{2}, \quad r_{1}, r_{2} \in\left(\frac{1}{R}, 1\right) .
\end{gathered}
$$

По условию леммы сушествует постоянная $M>0$ такая, что для всех $r \in(1 / R, 1)$

$$
\int_{0}^{2 \pi}\left(\operatorname{Re} G_{1}\left(r e^{i \varphi}\right)\right)^{2} d \varphi=2 \pi a_{0}^{\prime 2}+\pi \sum_{k=1}^{\infty}\left(a_{k}^{\prime 2}+a_{k}^{\prime \prime 2}\right) r^{2 k} \leqslant M .
$$


Следовательно, для всех целых $N \geqslant 1$ и всех $r \in(1 / R, 1)$

$$
\sum_{k=1}^{N}\left(a_{k}^{\prime 2}+a_{k}^{\prime \prime 2}\right) r^{2 k} \leqslant M
$$

откуда (после перехода к пределу при $r \rightarrow 1-0$ в последнем неравенстве) имеем справедливое для всех $N \geqslant 1$ неравенство

$$
\sum_{k=1}^{N}\left(a_{k}^{\prime 2}+a_{k}^{\prime 2}\right) \leqslant M
$$

и, тем самым, получаем сходимость числового ряда

$$
\sum_{k=1}^{\infty}\left(a_{k}^{\prime 2}+a_{k}^{\prime \prime 2}\right)
$$

Поэтому ряд, стоящий в правой части равенства $(26)$, сходится равномерно по $r_{1} \in$ $(1 / R, 1), r_{2} \in(1 / R, 1)$. Переходя в этом равенстве к пределу при $r_{1} \rightarrow 1-0$, $r_{2} \rightarrow 1-0$, получаем соотношение

$$
\int_{0}^{2 \pi}\left(\operatorname{Re} G_{1}\left(r_{1} e^{i \varphi}\right)-\operatorname{Re} G_{1}\left(r_{2} e^{i \varphi}\right)\right)^{2} d \varphi \rightarrow 0, \quad r_{1} \rightarrow 1-0, \quad r_{2} \rightarrow 1-0
$$

из которого вытекает утверждение леммы.

ДОКАЗАТЕЛЬСТВо ЛЕММЫ 3 . Поскольку по условию множество функций (15) $L_{2}(0,2 \pi)$-ограничено, то для доказательства леммы достаточно установить, что при любом (целом) $k \geqslant 1$

$$
\begin{aligned}
& I_{k}(r)=\frac{1}{\pi} \int_{0}^{2 \pi} \operatorname{Re}\left(A\left(r e^{i \varphi}\right) G_{2}\left(r e^{i \varphi}\right)\right) \cos k \varphi d \varphi \rightarrow 0, \quad r \rightarrow 1-0, \\
& J_{k}(r)=\frac{1}{\pi} \int_{0}^{2 \pi} \operatorname{Re}\left(A\left(r e^{i \varphi}\right) G_{2}\left(r e^{i \varphi}\right)\right) \sin k \varphi d \varphi \rightarrow 0, \quad r \rightarrow 1-0,
\end{aligned}
$$

И

$$
I_{0}(r)=\frac{1}{2 \pi} \int_{0}^{2 \pi} \operatorname{Re}\left(A\left(r e^{i \varphi}\right) G_{2}\left(r e^{i \varphi}\right)\right) d \varphi \rightarrow 0, \quad r \rightarrow 1-0 .
$$

Из равенства (22) следует, что

$$
\begin{aligned}
I_{k}(r)= & \left(r^{2}-1\right)\left(\sum_{m=1}^{\infty} \lambda_{m k}^{\prime} \sum_{s=0}^{m-1} r^{2 m+k-2-2 s}\right. \\
& \left.+\sum_{m=k}^{\infty} \mu_{m k}^{\prime} \sum_{s=0}^{m-1} r^{2 m-k-2-2 s}\right), \quad k \geqslant 1
\end{aligned}
$$




$$
\begin{aligned}
J_{k}(r)= & \left(1-r^{2}\right)\left(\sum_{m=1}^{\infty} \lambda_{m k}^{\prime \prime} \sum_{s=0}^{m-1} r^{2 m+k-2-2 s}\right. \\
& \left.-\sum_{m=k}^{\infty} \mu_{m k}^{\prime \prime} \sum_{s=0}^{m-1} r^{2 m-k-2-2 s}\right), \quad k \geqslant 1, \\
I_{0}(r)= & \left(r^{2}-1\right) \sum_{m=1}^{\infty} \lambda_{m 0}^{\prime} \sum_{s=0}^{\infty} r^{2 m-2-2 s} .
\end{aligned}
$$

В силу (24) и (25) при $k \geqslant 1$ имеем неравенство

$$
\left|I_{k}(r)\right| \leqslant\left(1-r^{2}\right)\left(C R^{2 k}+C R^{k}\right),
$$

из которого следует соотношение $\left(27^{\prime}\right)$. Соотношения $\left(27^{\prime \prime}\right)$ и $\left(27^{\prime \prime \prime}\right)$ аналогично вытекают из неравенств $\left(24^{\prime}\right),\left(25^{\prime}\right)$ и $\left(24^{\prime \prime}\right)$. Лемма доказана.

Лемма 4. Семейство функиий $(10)\left(r_{0}=1 / R\right) L_{2}(0,2 \pi)$-компактно тогда и только тогда, когда $L_{2}(0,2 \pi)$-компактны каждое из семейств (14) и (15).

ДокАЗАТЕЛьство. Эта лемма, так же как и лемма 1 , показьвает определенную независимость в смысле $L_{2}(0,2 \pi)$-компактности слагаемых правой части равенства $(12)$ : из $L_{2}(0,2 \pi)$-компактности суммы вытекает $L_{2}(0,2 \pi)$-компактность каждого слагаемого.

Так как множество функций (13), связанное с первым слагаемым правой части равенства (12) и имеющее $L_{2}(0,2 \pi)$-предел при $r \rightarrow 1-0$, является $L_{2}(0,2 \pi)$-компактным, то в доказательстве нуждается лишш утверждение о необходимости. Если множество функций (10) $L_{2}(0,2 \pi)$-компактно, то из лемм 1 и 2 вытекает $L_{2}(0,2 \pi)$-компактность множества функций $(14)$, а следовательно, в силу равенства $(12)$ и $L_{2}(0,2 \pi)$-компактности множества функций $(13)$ и $L_{2}(0,2 \pi)$-компактность множества функций (15). Лемма доказана.

ДоКАЗАТЕЛЬСТВо ТЕОРЕМЫ $1^{\prime}$. Поскольку утверждение теоремы о необходимости тривиально, то мы останавливаемся лишь на доказательстве утверждения о достаточности. Из $L_{2}(0,2 \pi)$-компактности множества функций $(10)\left(r_{0}=1 / R\right)$ в силу лемм 4 и 2 вытекает сушествование $L_{2}(0,2 \pi)$-предела при $r \rightarrow 1-0$ множества функций (14), а в силу лемм 4,1 и 3 вытекает $L_{2}(0,2 \pi)$-компактность семейства (15) и его слабая $L_{2}(0,2 \pi)$-сходимость к нулю при $r \rightarrow 1-0$. Покажем, что тогда семейство функций (15) сходится к нулю при $r \rightarrow 1-0$ и в $L_{2}(0,2 \pi)$.

Прежде всего отметим, что если какая-либо последовательность функций семейства (15), для которой $r \rightarrow 1-0$, имеет $L_{2}(0,2 \pi)$-предел, то этот предел в силу слабой сходимости семейства $(15)$ к нулю непременно нулевой.

Если бы множество функций (15) не сходилось в $L_{2}(0,2 \pi)$ к нулю, то существовало бы $\varepsilon^{\prime}>0$ такое, что для любого $n \geqslant 1$ нашлось бы $r_{n}, 1 / n<r_{n}<1$, такое, что

$$
\left\|\operatorname{Re}\left(A\left(r_{n} e^{i \varphi}\right) G_{2}\left(r_{n} e^{i \varphi}\right)\right)\right\| \geqslant \varepsilon^{\prime}, \quad n=1,2, \ldots,
$$

при этом последовательность $\left\{r_{n}, n=1,2, \ldots\right\}$ можно считать монотонно возрастаюшей. В силу $L_{2}(0,2 \pi)$-компактности последовательности функций

$$
\left\{\operatorname{Re}\left(A\left(r_{n} e^{i \varphi}\right) G_{2}\left(r_{n} e^{i \varphi}\right)\right), 0 \leqslant \varphi \leqslant 2 \pi, n=1,2, \ldots\right\},
$$


в ней есть $L_{2}(0,2 \pi)$-сходящаяся подпоследовательность, сходящаяся, как указано вьше, к нулю. А это противоречит неравенству (28). Теорема доказана.

ДокАЗАТЕЛЬСТво тЕОРЕмы $2^{\prime}$. Здесь, так же как и в случае теоремы $1^{\prime}$, мы ограничиваемся лишь доказательством достаточности, поскольку утверждение о необходимости тривиально. Из $L_{2}(0,2 \pi)$-ограниченности (слабой $L_{2}(0,2 \pi)$-компактности) семейства функций (10) по лемме 1 следует $L_{2}(0,2 \pi)$-ограниченность как семейства функций (14), так и семейства (15). В силу леммы 2 из $L_{2}(0,2 \pi)$ ограниченности множества функций (14) вытекает его $L_{2}(0,2 \pi)$-сходимость (и тем более слабая $L_{2}(0,2 \pi)$-сходимость $)$ при $r \rightarrow 1-0$. В силу леммы 3 из $L_{2}(0,2 \pi)$ ограниченности семейства функций (15) вытекает его слабая $L_{2}(0,2 \pi)$-сходимость к нулю. Так как множество функций (13) также сходится в $L_{2}(0,2 \pi)$ при $r \rightarrow 1$, то согласно равенству (12) семейство функций (10) слабо $L_{2}(0,2 \pi)$-сходится. Теорема доказана.

\section{Список литературы}

1. Михайлов В. П. О существовании предельных значений решений полигармонического уравнения на границе области // Матем. сб. 1996. Т. 187. №11. С. 89-114.

2. Михайлов В. П. Существование граничного значения у метагармонических функций // Матем. сб. 1999. Т. 190. № 10. С. 17-48.

3. Riesz F. Über die Randwerte eines Analytische Funktion // Math. Z. 1923. V. 18. P. 87-95.

4. Привалов И. И. Граничные свойства однозначных аналитических функций. М.: ГИТТЛ, 1950.

5. Михайлов В. П. О граничных значениях решений эллиптических уравнений в областях с гладкой границей // Матем. сб. 1976. Т. 101. № 2. С. 163-188.

6. Гущин А. К. О задаче Дирихле для эллиптического уравнения второго порядка // Матем. сб. 1988. Т. 137. № 1. С. 19-64.

7. Векуа И. Н. Новые методы решения эллиптических уравнений. М.-Л.: ГИТТЛ, 1948.

Математический институт

им. В.А. Стеклова РАН, г. Москва

Поступила в редакцию

15.03 .2004 